\title{
Expression of the zebrafish intermediate neurofilament Nestin in the developing nervous system and in neural proliferation zones at postembryonic stages Julia Mahler and Wolfgang Driever*
}

\author{
Address: Developmental Biology Department, Institute of Biology I, University of Freiburg Hauptstrasse 1, D-79104 Freiburg, Germany \\ Email: Julia Mahler - julia.mahler@biologie.uni-freibrug.de; Wolfgang Driever* - driever@biologie.uni-freiburg.de \\ * Corresponding author
}

Published: 25 July 2007

BMC Developmental Biology 2007, 7:89 doi:10.1186/147/-2/3X-7-89

This article is available from: http://www.biomedcentral.com/I47/-2/3X/7/89

(c) 2007 Mahler and Driever; licensee BioMed Central Ltd.

This is an Open Access article distributed under the terms of the Creative Commons Attribution License (http://creativecommons.org/licenses/by/2.0), which permits unrestricted use, distribution, and reproduction in any medium, provided the original work is properly cited.
Received: 20 March 2007

Accepted: 25 July 2007

\begin{abstract}
Background: The intermediate filament Nestin has been reported as a marker for stem cells and specific precursor cell populations in the developing mammalian central nervous system (CNS). Nestin expressing precursors may give rise to neurons and glia. Mouse nestin expression starts at the onset of neurulation in the neuroectodermal cells and is dramatically down regulated when progenitor cells differentiate and become postmitotic. It has been reported that in the adult zebrafish (Danio rerio) active neurogenesis continues in all major subdivisions of the CNS, however few markers for zebrafish precursors cells are known, and Nestin has not been described in zebrafish.
\end{abstract}

Results: We cloned a zebrafish nestin gDNA fragment in order to find a marker for precursor cells in the developing and postembryonic brain. Phylogenetic tree analysis reveals that this zebrafish ortholog clusters with Nestin sequences from other vertebrates but not with other intermediate filament proteins. We analyzed nestin expression from gastrula stage to 4 day larvae, and in postembryonic brains. We found broad expression in the neuroectoderm during somitogenesis. In the larvae, nestin expression progressively becomes restricted to all previously described proliferative zones of the developing and postembryonic central nervous system. nestin expressing cells of the forebrain also express PCNA during late embryogenesis, identifying them as proliferating precursor or neural stem cells. nestin is also expressed in the cranial ganglia, in mesodermal precursors of muscle cells, and in cranial mesenchymal tissue.

Conclusion: Our data demonstrate that in zebrafish, like in mammals, the expression of the intermediated neurofilament nestin gene may serve as a marker for stem cells and proliferating precursors in the developing embryonic nervous system as well as in the postembryonic brain.

\section{Background}

The intermediate filament Nestin has been reported as a marker for stem cells as well as precursor populations of specific cell types in the developing mammalian central nervous system (CNS) giving rise to both neurons and glia
$[1,2]$. Nestin is classified as type IV neurofilament, which together with microfilaments and microtubules constitute a major component in the cytoskeleton. In contrast to other more general cytoskeletal elements, intermediate filaments are expressed in a cell type specific manner, and 
major differentiation steps are marked by the transition from one intermediate filament type to another. With the onset of neurulation neuroectodermal cells start to express nestin. The expression is dramatically down regulated when progenitor cells differentiate and become postmitotic [2-4], reviewed in [5].

nestin mRNA also has been reported to be expressed in the developing myotome [6] and skeleton muscle precursors $[2,6]$, as well as in mesenchymal pancreatic cells [7], the intestine [8], and cranial ganglia [9].

While Nestin has been investigated extensively in mammalian systems, including rat [2], mouse [10], and human [11], as well as in chick [12], it has not been reported for fish so far. Zebrafish have evolved as a genetic and experimental model organism that is ideally suited to study basic principles of neural development [13-15]. It has been reported that in the adult zebrafish active neurogenesis continues in all major subdivisions of the CNS [16]. Thus, zebrafish serve as an excellent model for studying neural stem cells and neural regeneration. In detailed BrdU incorporation studies, distinct proliferation zones have been identified in all subdomains of the zebrafish brain along the rostrocaudal CNS axis [16-18]. It appears that, like in mammals, neuroblasts are also continuously generated in neurogenic niches in the subependymal/subventricular regions of the brain.

While BrdU incorporation studies may identify neural stem cells, these experiments are not easily integrated into most experimental setups. Other markers used to identify zebrafish neural stem cells include: PCNA [19], MCM5 $[15,20]$, and anti-phospho-histone antibodies. However, these markers are not restricted to neural proliferating cells. To identify a specific marker for neural stem cells and precursors, we cloned and analyzed the expression of a zebrafish Nestin ortholog.

\section{Results}

\section{A zebrafish nestin ortholog}

In order to find a marker for precursor cells in the developing zebrafish brain we analyzed nestin (nes) homologous genes in zebrafish. We compared nestin sequences from various different species and performed a blast search of zebrafish genomic sequences to identify whether there are duplicated zebrafish nestin genes. We identified a single nestin ortholog with the ENSEMBL gene number ENSDAR00000040236 [21]. We compared the predicted mRNA as well as other zebrafish intermediate filaments with protein sequences from other species. Phylogenetic tree analysis revealed that the predicted zebrafish Nestin protein clusters with mouse, human, and chicken Nestin, but not with the other intermediated neurofilaments (Fig. 1). Using PCR on genomic DNA we cloned a 704 bp nestin fragment covering the second exon, we generated antisense probes, and investigated nestin expression in the developing zebrafish brain.

\section{Expression of nestin during zebrafish somitogenesis and in neuronal tissues}

We assayed the nestin expression pattern by in situ hybridization with an antisense RNA probe in zebrafish embryos and larvae staged from $60 \%$ epiboly up to 96 hours post fertilization (hpf). We also determined nestin expression in sections of the post-embryonic zebrafish brain at 28 dpf.

nestin expression was not detected before the 3 somite stage (10-11 hpf) (Fig. 2A,B). Earliest expression of nestin was detected during mid-neurulation (Fig. 2C-F"). At this stage nestin is expressed in a domain laterally adjacent to midline cells. Control embryos processed with a sense RNA for nestin show no obvious staining at this stage (Fig. 2D). Serial sections reveal that adaxial mesoderm cells express nestin in the trunk, and in the posterior trunk nestin is expressed in the neural plate (Fig. 2F-F"). At 18 somite stage nestin is also expressed in premigratory and migrating neural crest cells (data not shown).

At $24 \mathrm{hpf}$ nestin is widely expressed throughout the developing nervous system (Fig. 2G). A similar widespread expression has been reported for comparable stages of mouse development $[1,3]$. Zebrafish nestin expression is restricted to more defined regions of the CNS as the embryo further develops. We will now first describe the non-neuronal expression domains and later focus on the CNS expression.

Outside of the nervous system, mammalian nestin has been reported to be expressed in head mesenchyme and muscle precursors [6]. In contrast to nestin expression in mouse, zebrafish nestin was detected neither in developing somites nor in the myotome of mature somites. In fin

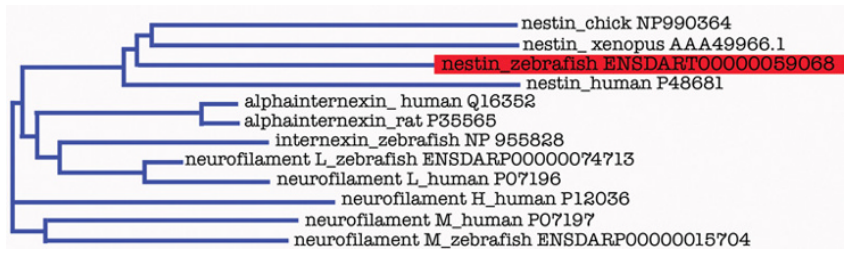

Figure I

Phylogenic analysis of nestin protein. The predicted nestin sequence (Ensembl) was translated into protein sequence and compared with other nestin proteins and intermediated neurofilaments of human, frog, chick, rat, and zebrafish. The analysis was performed with VectorNTI software (Neighbor joining method). For each protein either the NCBI, SwissProt or Ensembl ID numbers are noted. 


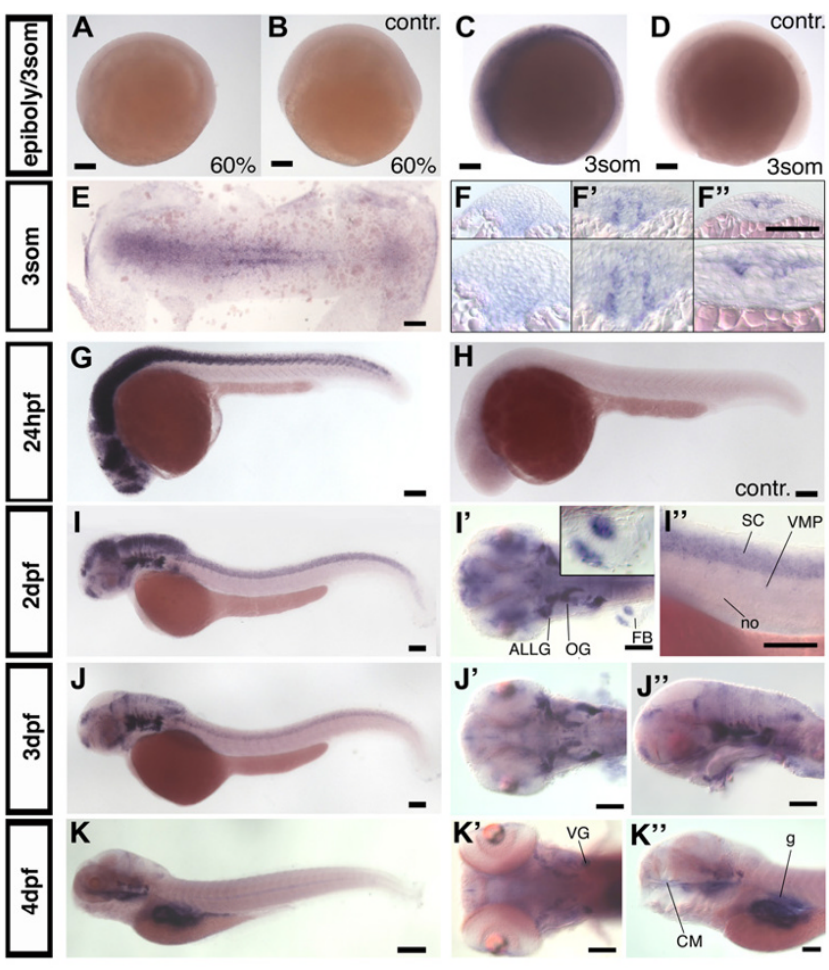

Figure 2

nestin expression in zebrafish development. (A-D): nestin expression pattern during gastrula and early somitogenesis stages, (A) nestin is not expressed at $60 \%$ epibloy stage, (B) sense control. (C, D): First expression is detectable at 3 somite stage. (E, F-F"): Flat mount and cross sections of a 3 somite embryo. ( $G$, sense control in $H$ ): Lateral view of 24 hpf embryo, nestin is expressed widely in CNS. (I, I'): $2 \mathrm{dpf}$, lateral view (I), dorsal view (I') nestin is expressed in head ganglia and fin buds; insert in ( $\left.I^{\prime}\right)$ shows higher magnification of a fin bud; (I") higher magnification of the spinal cord. nestin expression in the ventral root may correlate with glia or neuroblasts. (J-J"): At $3 \mathrm{dpf}$ nestin expression becomes more restricted to proliferation zones, $(J)$ lateral view, (J', J") higher magnifications, dorsal ( $\mathrm{J}^{\prime}$ ) and lateral (J") view. (K-K"): 4 dpf: nestin expression in the CNS is almost completely restricted to proliferation zones. Further expression is detected in the cranial ganglia, the gut and the craniofacial mesenchyme ( $\mathrm{K} ")$. Abbreviations: ALLG: anterior lateral line ganglion; $\mathrm{cm}$ : craniofacial mesenchyme; FB: fin bud; g: gut; OG: octaval ganglion; no: notochord; SC: spinal cord; VG: vagal ganglion; VMP: ventral motoneuron precursors. (A-E, G-K"): anterior left, animal pole up, (F-F"): cross sections, dorsal up. Scale bars: 100 $\mu \mathrm{m}$, except K: scale bar: $200 \mu \mathrm{m}$.

buds nestin is expressed at $2 \mathrm{dpf}$ in two stripes of mesoderm which will give rise to muscle tissue (Fig. 2I'). Further, from $4 \mathrm{dpf}$ on nestin is expressed in craniofacial mesenchyme adjacent to the ethmoid plate (eth) and palatoquadrate (pq) (Fig. 2K"; Fig. 4F,I,S) and weakly in epithelial cells of the gut (Fig. 2K"; Fig. 4Q) [8].

\section{Expression of nestin in the central nervous system (CNS)}

At $24 \mathrm{hpf}$ nestin is expressed widely throughout the developing nervous system. Comparing nestin expression at 2 dpf, $3 \mathrm{dpf}$, and $4 \mathrm{dpf}$ in zebrafish embryos reveals that nestin expression becomes progressively restricted to domains where the progenitor cell pools have been identified [18]: Ventricular zones (VZ) in the telencephalon (TC) and diencephalon (DC), the midbrain hindbrain boundary (MHB), and the ciliary marginal zone (CMZ) of the retina (Fig. $2 \mathrm{G}, \mathrm{I}, \mathrm{J}, \mathrm{K}$ ). From $2 \mathrm{dpf}$ to $4 \mathrm{dpf}$, in the TC and DC, nestin expression starts to be restricted to the ventricular zones in the region of the subpallium and the ventral DC (Fig. 3A-I;). At 2 dpf nestin expression in the ventricular region of the DC is difficult to discern in whole mount ISH embryos because of the broad expression domain in the pretectal area (Fig. 3G). At $4 \mathrm{dpf}$ the expression in the DC becomes more distinct (Fig. 3F). The pretectal nestin expression domain becomes also more restricted with further development of the nervous system and at $4 \mathrm{dpf}$ it is restricted to very faint expression in a domain close to the ventricle (Fig. 3C). In the retina nestin expressing cells are initially detected in a broad zone including the ganglion cell layer (Fig. 3M-O). Similar to the diencephalon, nestin expression in the retina continuously regresses and by $4 \mathrm{dpf}$ becomes restricted to the ciliary marginal zone, which constitutes the retinal proliferation zone [22,23] (Fig. 3O). Further posterior in the area of the MHB, the distribution of nestin positive cells also becomes more localized to the ventricle walls (Fig. 3G,H,I) from $1 \mathrm{dpf}$ to $4 \mathrm{dpf}$. The MHB is clearly lined by a row of nestin expressing cells at $3 \mathrm{dpf}$ (Fig. 3K). In the hindbrain initially broad nestin expression becomes restricted to cell populations adjacent to the ventricle and to some dorsal areas. Expression in the cerebellum also progressively decreases (Fig. 2J,J"; Fig. 3A-C).

Further we performed sections through $2 \mathrm{dpf}$ and $4 \mathrm{dpf}$ embryos to analyze in more detail the nestin expression pattern especially in regions, which were difficult to examine by transmitted light microscopy of whole-mount in situ hybridized embryos. In the TC nestin expression is restricted to ventricular zones (Fig. 4A). From $2 \mathrm{dpf}$ to 4 dpf nestin expression in the area of the dorsal TC and the pretectal area is strongly down regulated (Fig. 4A-F). In the DC cells close to the ventricle express nestin predominantly in the dorsal part of the ventricular region of the DC (Fig. 4D,E). In parts of the intermediate hypothalamus nestin is expressed at $4 \mathrm{dpf}(\mathrm{Fig} 4 \mathrm{~N}, \mathrm{O})$. Transversal sections at the level of the MHB through $2 \mathrm{dpf}$ and $4 \mathrm{dpf}$ zebrafish embryos revealed a strong nestin expression in this area (Fig. 4G-I) and correlates with regions of ongoing active neurogenesis [24]. Further, in the HB a cluster of cells bilaterally adjacent to the midline expresses nestin (Fig. 2I'; Fig. 4J). At 4 dpf expression in the hindbrain is almost completely absent, except for some scattered nestin 
expressing cells in the medulla oblongata (Fig. 4P). Sections as well as whole mount ISH revealed, that nestin is expressed throughout the spinal cord, except for the floorplate (Fig. 2I,I"; Fig. 4M). Zimmerman and colleagues [6] report that in the nestin:lacZ transgenic mice nestin:LacZ is not expressed in the floorplate and ventral midline. nestin mRNA is detectable in the ventral root of the spinal cord (Fig. 2I"). Mammalian motor neurons do not express nestin [6], but an antibody against Nestin protein (rat401) stains rat ventral root before axon outgrowth [1].

\section{Expression of nestin in the peripheral nervous system (PNS)}

nestin in mammals is strongly expressed in ganglia of the posterior cranial nerves [9]. We also find strong expression at $2 \mathrm{dpf}$ and $3 \mathrm{dpf}$ in the octaval ganglion (ganglia of

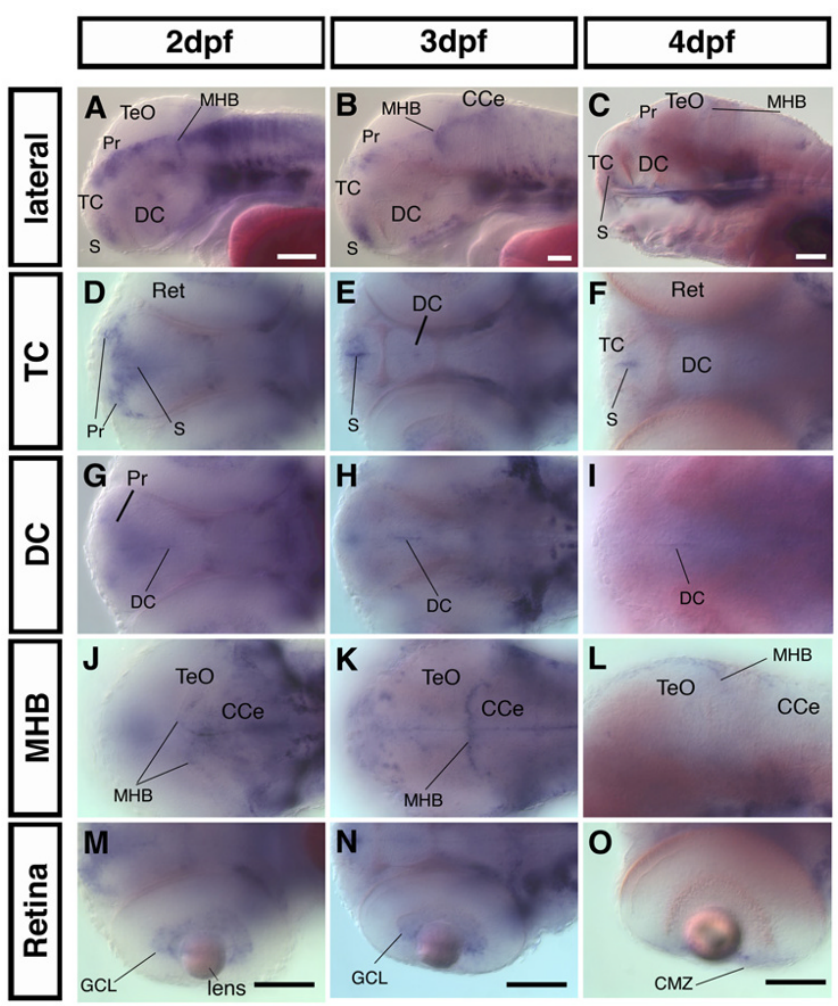

Figure 3

In the developing CNS nestin expression becomes progressively restricted to proliferative zones. Analysis of nestin expression in the head by whole mount in situ hybridization. (A-C): lateral views, anterior left, dorsal up, eyes were removed for better visualization of the expression pattern in the DC. A mid-saggital focal plane is shown (D-O, except $L$ ): dorsal views, anterior left, the dorsoventral level of the focal plane is indicated at left. (L): lateral view. Abbreviations: CCe: Cerebellum; CMZ: ciliary marginal zone; DC: diencephalon; GCL: ganglion cell layer; MHB: midbrain hindbrain boundary; Pr: pretectum; Ret: retina; S: subpallium: TC: telencephalon; $\mathrm{TeO}$ : optic tectum. Scale bars: $100 \mu \mathrm{m}$.
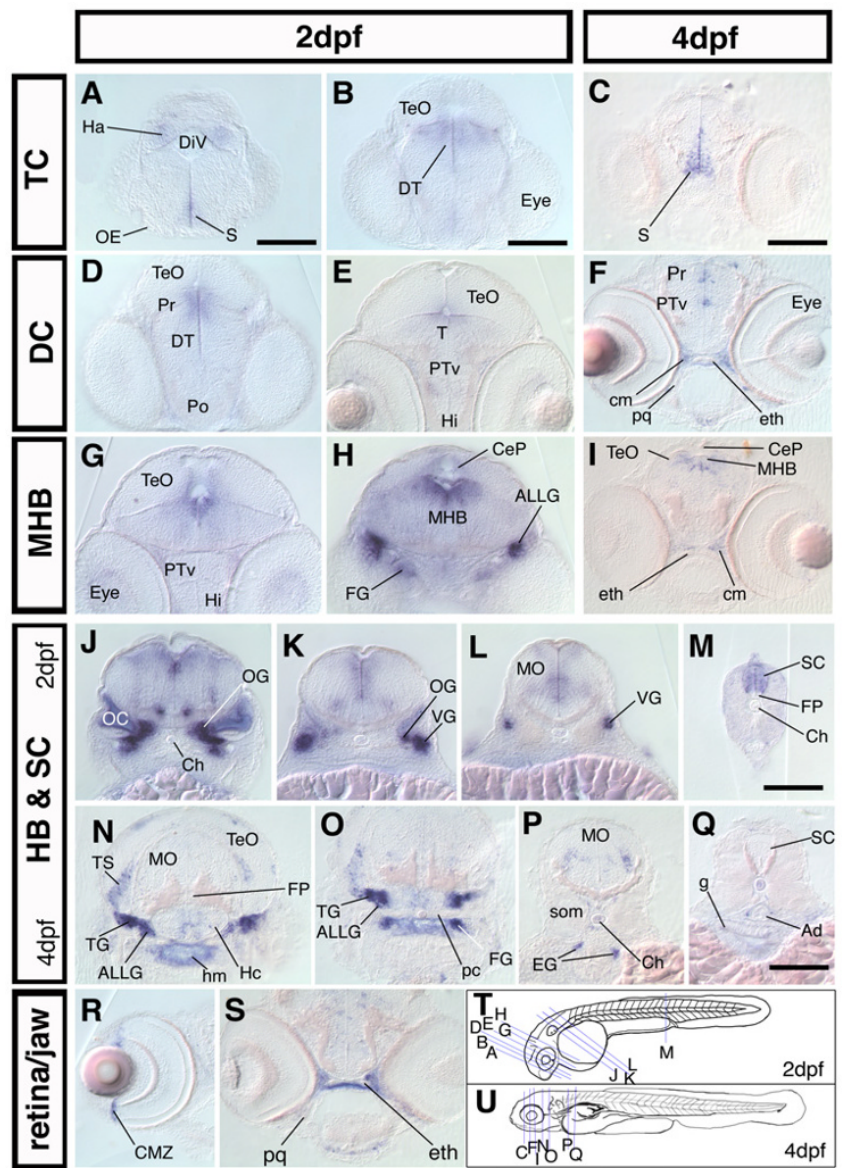

Figure 4

nestin is expressed in proliferative zones during the development of the CNS. Cross sections of $2 \mathrm{dpf}$ and $4 \mathrm{dpf}$ embryos; dorsal up. (A-S): frontal and transversal cross section of nestin expression along the rostrocaudal axis of the brain and in the spinal cord reveals expression in major proliferative zones The relative rostrocaudal levels of each section are indicated in $T$ and $U$. (A-I): sections at levels of forebrain and midbrain as well as midbrain-hindbrain boundary. ( $-\mathrm{Q}$ ): sections at the level of $\mathrm{HB}$ and $\mathrm{SC}$, showing nestin expression in the cranial ganglia and the $\mathrm{HB}$, $\mathrm{MO}, \mathrm{SC}$. (R): cross section through the eye of a $4 \mathrm{dpf}$ embryo, nestin is expressed in the CMZ; (S): cross section of a 4 dpf embryo at the level of the ethmoid plate, nestin is expressed in the craniofacial mesenchyme adjacent to developing cartilage tissue. $(T, U)$ : scheme of a $2 \mathrm{dpf}(\mathrm{T})$ and a $4 \mathrm{dpf}(\mathrm{U})$ zebrafish embryo, the levels of the cross sections shown in A-S are indicated by blue lines. Abbreviations: Ad: Aorta dorsalis; ALLG: anterior lateral line ganglion; CeP: cerebellar plate; Ch: chorda dorsalis; $\mathrm{cm}$ : craniofacial mesenchyme; CMZ: ciliary marginal zone; DiV: diencephalic ventricle; DT: dorsal thalamus; eg: enteric ganglia; eth: ethmoid plate; FG: facial ganglion; FP: Floor plate; g: gut; Ha: habenula; Hc: caudal hypothalamus; $\mathrm{Hi}$ i intermediate hypothalamus; hm: head mesenchyme; MHB: midbrain hindbrain boundary; MO: medulla oblongata; OC: otic capsule; OE: olfactory epithelium; OG: octaval ganglion; pc: parachordal cartilage; pq: palatoquadrate; Po: preoptic region; Pr: pretectum; PTv: ventral part of the posterior tuberculum; S: subpallium; SC: spinal cord; som: somites; T: midbrain tegmentum; TeO: optic tectum; TG: trigeminal ganglion; TS: torus semicircularis; VG: vagal ganglion. Scale bars: $100 \mu \mathrm{m}$. 
the n. octavus, VIII, vestibulocochlear nerve) (Fig. 2I'; Fig. $4 \mathrm{~J}, \mathrm{~K})$, the anterior and posterior part of the lateral line ganglia (associated with parts of VIIIth ganglion) (Fig. 2I'; Fig. $4 \mathrm{H}$ ), the facial ganglia (n. facialis, VII) (Fig. $4 \mathrm{H}$ ), and the ganglia of the nervus vagus $(\mathrm{X})(\mathrm{Fig} .4 \mathrm{~K}, \mathrm{~L})$. The ganglia of the nervus vagus and the lateral line ganglia at this stage may contain a mixture of already differentiated cells and progenitor cells, with the latter situated more proximal to the midline than the differentiated cells. At $3 \mathrm{dpf}$ nestin is still broadly expressed in cells of cranial ganglia (Fig. 2J). At $4 \mathrm{dpf}$ the expression appears reduced (Fig. $2 \mathrm{~K}^{\prime}, \mathrm{K}^{\prime \prime}$ ), but transversal sections through the head show that at $4 \mathrm{dpf}$ nestin is still expressed in the trigeminal (V) ganglion, the octaval and lateral line ganglia, and the vagal ganglion (Fig. 4N,O), and data not shown). In the torus semicircularis (TS), a sensory nucleus which is the mesencephalic target of the octavolateralis-system [25], nestin expression can also be detected (Fig. 4N,O). Similar to mammals [8], at $4 \mathrm{dpf}$ nestin expression is also detected in enteric ganglia of the peripheral nervous system (PNS) (Fig. 4P).

\section{nestin expressing cells co-express pcna in the forebrain during late embryogenesis}

Nestin has been reported as a marker for precursor populations of specific cell types in the developing mammalian CNS $[1,2]$. The expression of nestin in cells close to the ventricle throughout the CNS led to the assumption that also in zebrafish nestin is a marker for proliferating precursor cells. Therefore, we performed double fluorescent in situ hybridization to detect nestin and proliferating cell nuclear antigen (PCNA) expression to investigate whether pcna positive cells also co-express nestin in zones of proliferation. At $2 \mathrm{dpf}$ we could detect areas with cells expressing both nestin and pcna in the proliferation zones of the forebrain (Fig. 5A-C). Cells expressing nestin and pcna are located along the TC ventricle (Fig. 5D-F) and in the ventricle walls of the ventral DC (Fig. 5G-I). Further, at $3 \mathrm{dpf}$ co-expression was detectable in the subventricular zone of the TC (Fig. 5J-L). Thus, nestin expression appears to correlate with stem cell and precursor territories in the forebrain proliferation zones. However, expression domains of nestin in the peripheral ganglia do not correlate with pcna expression (data not shown), confirming that nestin is also expressed in postmitotic or differentiated cells in the peripheral nervous system.

\section{nestin expression in post-embryonic stem cell zones and migrating precursors}

In order to investigate nestin expression in the post-embryonic zebrafish brain we examined nestin mRNA distribution in $28 \mathrm{dpf}$ zebrafish brain. We performed whole brain ISH and generated serial transversal sections (50 micrometer) along the rostrocaudal neural axis. We use the neuroanatomical terms for the adult zebrafish according to the zebrafish brain atlas by Wullimann and colleagues [25]. In these cases where we use the names of the ana-

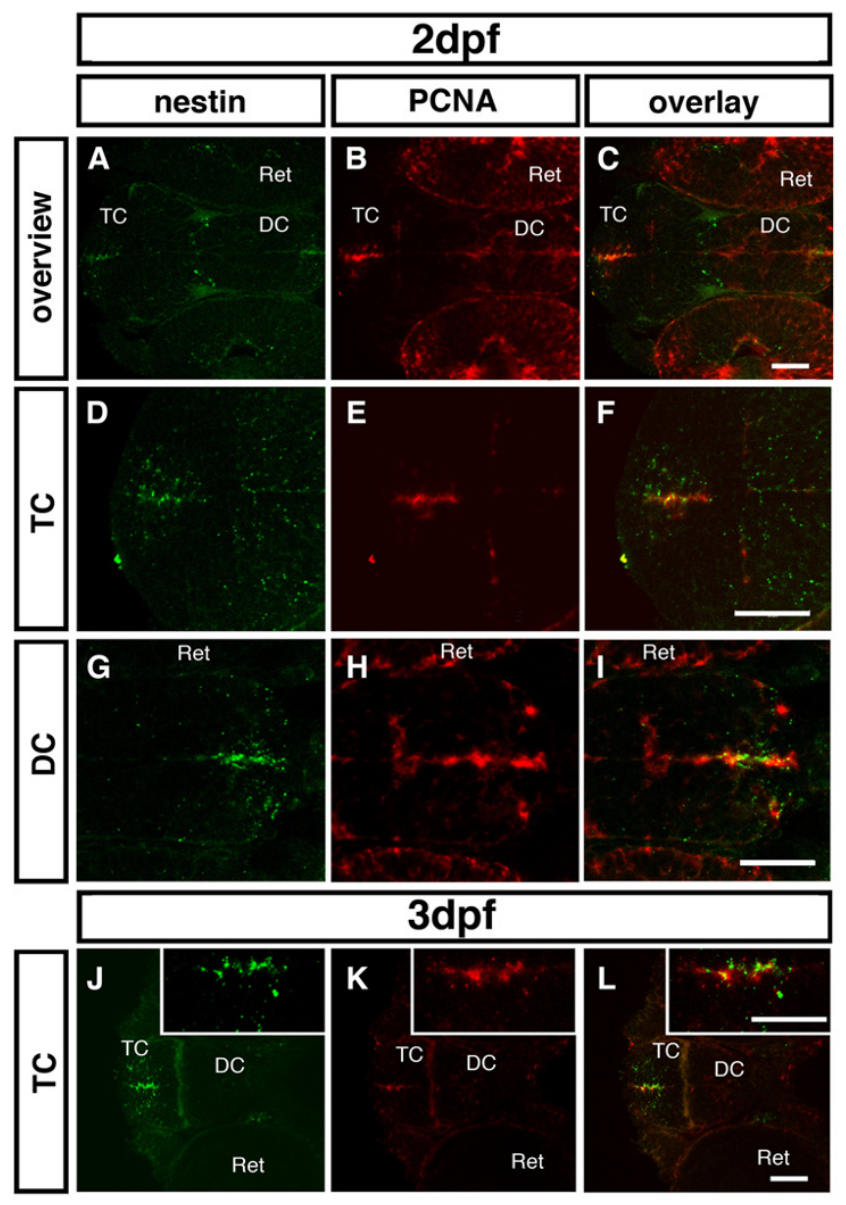

Figure 5

Co-expression of nestin and pcna in the zebrafish

forebrain. Double fluorescent ISH was performed for $2 \mathrm{dpf}$ $(\mathrm{A}-\mathrm{I})$ and $3 \mathrm{dpf}(\mathrm{J}-\mathrm{L})$ zebrafish embryos. nestin is shown in green (A, D, G, J); pcna in red (B, E, H, K); overlay (C, F, I, L). Inserted pictures in (J-L) show a higher magnification of the boxed areas. (A-C): Overview over the proliferation zones in the $2 \mathrm{dpf}$ zebrafish forebrain. (D-F): Higher magnification of the ventricular zone of the TC shows co-expression. $(\mathrm{G}-\mathrm{H})$ : Higher magnification of the subventricular zone in the ventral DC reveals co-expression of nestin and pcna. (J-L): At $3 \mathrm{dpf}$ cells co-expressing nestin and pcna are detectable along the walls of the telencephalic ventricle. Abbreviations: DC: diencephalon; Ret: retina; TC: telencephalon. Scale bar: $50 \mu \mathrm{m}$.

tomical structures to describe localization of expression pattern, nestin is often expressed at the ventricular surface of the named anatomical structures.

\section{Telencephalon}

The most anterior expression of nestin we could detect was a weak staining in the posterior part of the olfactory bulb $(\mathrm{OB})$. Here nestin is expressed only in the ventral parts of the TC (V: ventral telencephalic area). Few nestin positive cells are detectable in the dorsal nucleus of the ventral tel- 
encephalic area $(\mathrm{Vd})$ close to the telencephalic ventricle (TelV) (Fig. 6A). Throughout the TC nestin positive cells accumulate in the SVZ of Vd and Vv (ventral nucleus of V). Anterior to the anterior commissure (AC) nestin is strongly expressed in cells of the $\mathrm{Vv}$ and $\mathrm{Vd}$, up to 5-10 cell diameters away from the ventricle surface (Fig. 6B). The expression is slightly reduced in the more posterior parts of the TC at the level of the AC. nestin is weakly expressed in the supracommissural and postcommissural nuclei (data not shown).

We also detected a weak expression of nestin in the ventral parts of the anterior parvocellular preoptic nucleus (PPa) (data not shown). At the level of the telencephalo-diencephalic boundary (preoptic region) nestin expression extends dorsally and is broadly expressed along the most anterior part of the diencephalic ventricle (DiV) in the area of ventral habenular nucleus (HaV), thalamic nuclei $(\mathrm{TN})$, in the posterior parvocellular preoptic nucleus (PPp), and ventral periventricular hypothalamus (Hv) (Fig. 6C).

\section{Diencephalon}

nestin is expressed all along the subventricular zone of the DiV. In the region of the optic tract (OT) speckled nestin expression was visible, which might be glia precursor cells migrating along the optic tract (Fig. 6D). Dorsally nestin is expressed in distinct ventricular zones: the ventral habenular nucleus (Hav), the periventricular pretectal nucleus (PP), the thalamic nuclei (TN) including the dorsal thalamic nucleus (DT) and the ventromedial thalamic nucleus (VM) (Fig. 6C,D). In the ventral DC nestin positive cells are detected in the region of the periventricular nucleus of the posterior tuberculum (TPp) and the posterior tuberal nucleus (PTN) (Fig. 6D,E). Strong nestin expression is detected in the cells adjacent to the lateral recess (LR) of the $\mathrm{DiV}$ in the area of the dorsal zone of the periventricular hypothalamus (Hd) (Fig. 6E,F) throughout its entire anterior-posterior extend (Fig. 6E-I). In the more posterior part of the ventral DC nestin expression is expressed at lower levels in the TPp and stronger in the ventral zone of the periventricular hypothalamus $(\mathrm{Hv})$ (Fig. 6F,G). In cells adjacent to the DiV nestin expression is also detectable in the most ventroposterior part of the DC, in the region of the caudal hypothalamus (Hc) (Fig. $6 \mathrm{H})$.

\section{Mesencephalon}

nestin is expressed in two zones of mesencepalon: in the optic tectum (TeO) and the torus longitudinalis (TL), which is ventrally attached to the tectum and reaches from its anterior to its posterior end [18] (Fig. 6E-G). nestin positive cells are located at the ventricle-contacting surface of the torus longitudinalis along its entire anterior-posterior extension in the mesencephalon (Fig. 6E-G). Further,

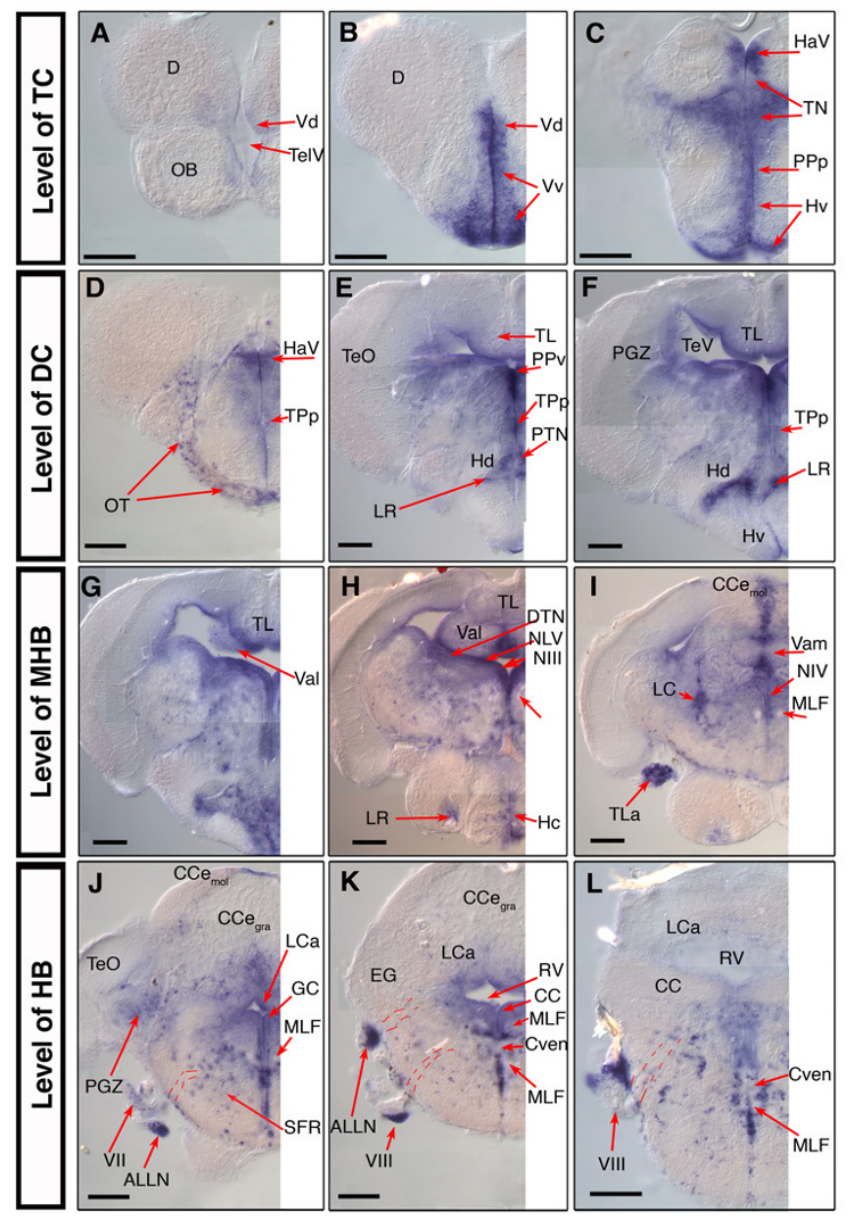

\section{Figure 6}

nestin expression in the juvenile $28 \mathrm{dpf}$ old zebrafish brain. Analysis of nestin expression in the brain of the $28 \mathrm{dpf}$ zebrafish by whole mount in situ hybridization using nestin anti sense mRNA probes on whole dissected brain. (A-L): selected $50 \mu \mathrm{m}$ cross sections from a serially sectioned brain, the rostrocaudal levels of the sections are indicated at left; dorsal up. Pictures show only the left half of the brain. Dashed lines in J, K, $L$ indicate the tracts of the cranial nerves. Abbreviations: ALLN: anterior lateral line nerve; CCe: corpus cerebelli; Cven: commissura ventralis rhombencephali; D: dorsal telencephalic area; DiV: diencephalic ventricle; EG: eminentia granularis; GC: griseum centrale; $\mathrm{HaV}$ : ventral habenular nucleus; $\mathrm{Hc}$, $\mathrm{Hd}$, $\mathrm{Hv}$ : caudal, dorsal, ventral zone of the periventricular hypothalamus; LC: locus coeruleus; LCa: locus caudalis cerebelli; LR: lateral recess of the DiV; MLF: medial longitudinal fascicle; NIII: oculomotor nucleus; NIV: trochlear nucleus; NMLF: nucleus of MLF; NLV: nucleus lateralis valvulae; OT: optic tract; PGZ: periventricular gray zone of the optic tectum; PPa, PPp: parvocellular preoptic nucleus, anterior, posterior part; PPv: periventricular pretectal nucleus, ventral part; PTN: posterior tuberal nucleus; RV: rhombencephalic ventricle; $\mathrm{TeO}$ : optic tectum; TeV: tectal ventricle; TelV: telencephalic ventricle; TL: Torus longitudinalis; TLa: torus lateralis; TN: thalamic nuclei; Val, Vam: lateral, medial division of valvula cerebelli; $\mathrm{Vd}, \mathrm{Vv}$ : dorsal, ventral nucleus of ventral telencephalic area; TPp: periventricular nucleus of the posterior tuberculum; III: oculomotor nerve; VII: facial nerve; VIII: octaval nerve; Scale bars: $100 \mu \mathrm{m}$. 
in the $\mathrm{TeO}$ cells of the ventricle contacting part of the periventricular gray zone (PGZ), express nestin mRNA in a relatively homogeneous manner around the ventricle. The expression domain spans the entire rostro-caudal axis of the PGZ. The more superficial layers of the $\mathrm{TeO}$ and the parts of the PGZ not contacting the ventricle do not express nestin. nestin is also strongly expressed in the dorsal tegmental nucleus (DTN) and the nucleus lateralis valvulae (NLV), two ventricle contacting structures of more ventral parts of the mesencephalon (Fig. 6H). In the most rostral part of the superior reticular formation, which also belongs to the mesencephalon, nestin expressing cells can be detected in a scattered manner (Fig. 6J).

\section{Hindbrain and midbrain hindbrain boundary (MHB)}

In teleosts the cerebellum can be subdivided in three parts $[16,25]$ : The corpus cerebelli (CCe), the vestibulolateralis lobe (consists of the medial caudal lobe (LCa) and the eminentiae granulares (EG) and the valvula cerebelli pars medialis and pars lateralis (Vam/Val). The midbrain hindbrain boundary (MHB) is known as a zone of continuous neurogenesis in the developing as well as in the mature brain. The MHB in the post-embryonic brain also contains nestin expressing cells. In the anterior part of the cerebellum nestin is strongly expressed along the midline, mainly in the molecular layer, the granular layer seems to be devoid of nestin expression cells. (Fig. 6H,I). nestin expressing cells can be found in both, medial and lateral, parts of the valvula. In the Val we could detect strong nestin expression in ventricle contacting regions. In the medial part of the valvula cerebelli (Vam) nestin is strongly expressed in the molecular as well as in the granular layer close to the tectal ventricle (Fig. 6G-I). In the eminentiae granulares nestin expressing cells are detectable. In the $\mathrm{HB}$ like in other parts of the brain, nestin expressing cells are located in the SVZ of the rhombencephalic ventricle. Here strong expression is detected in the ventricle contacting part of the caudal lobe of the cerebellum (LCa) (Fig. 6J-L). Further, in the HB more widely distributed nestin positive cell groups, e.g. in the granular and molecular layer of the caudal lobe, are detectable compared to the more anterior parts of the brain (Fig. 6JL).

\section{Cranial Ganglia Nerves}

In the post-embryonic brain as well as in the embryonic brain, nestin is expressed in the nuclei of cranial nerves. High expression of nestin is detected in the nucleus of the third cranial nerve (oculomotor nerve; NIII) (Fig. 6H). The axon-containing tract of the medial longitudinal fascicle (MLF), which carries axons descending to the spinal cord, is devoid of nestin expression throughout its rostrocaudal extend (Fig. 6H-L), as well as in the nucleus of the trochlear nerve (nervus trochlearis, IV) (Fig. 6I). Surprisingly, in the area of cranial nerves, which have already left the brain stem, we detected strong nestin expression. Analysis at higher resolution revealed that nestin is not expressed in the fascicles themselves but in clusters of cells, which appear to accompany the nerves. This was observed for the fascial nerve (n. fascialis, VII) as well as for the octaval nerve (n. octavus, VIII) and the associated anterior lateral line nerve (ALLN), and led us to speculate that these cells might be glia or migrating glia precursors, similar to findings in mammalian systems [26] (Fig. $6 \mathrm{G}, \mathrm{J}, \mathrm{K}, \mathrm{L})$.

\section{Discussion and Conclusion}

In mammals the intermediate neurofilament Nestin is a well established marker for neuronal stem cells and proliferating precursor cell populations, as well as for precursor cell populations in some other tissues (reviewed in [5]). Previously, nestin expression has only been characterized in mammalian systems $[2,10,11]$, and chick [12]. However, while a gene prediction for a zebrafish nestin has been placed by ENSEMBL on linkage group 16 at 27.11 $\mathrm{Mb}$, until now it has not been demonstrated that this intermediate filament represents a true nestin ortholog in teleosts. Here we provide two lines of evidence that this zebrafish intermediate filament is the true nestin ortholog: (1) Zebrafish Nestin protein sequence clusters with higher vertebrate Nestin proteins, and is clearly separated from other vertebrate intermediate neurofilaments, based on phylogenetic tree analysis. (2) nestin is expressed at embryonic, larval, and juvenile stages in cell populations which in the CNS largely represent stem and precursor cells. Subsets of nestin positive cells co-express the proliferating cell nuclear antigen PCNA - zebrafish nestin expression thus correlates with the expression pattern described in mouse [4].

We found that outside of the nervous system nestin is expressed in mesodermal muscle precursor cells and in craniofacial mesenchyme. This correlates with nestin expression in mammals at comparable developmental stages, which has been reported for head mesenchyme and muscle precursors [6]. In contrast to the reported nestin expression in mouse, we could not detect nestin expression neither in developing somites nor in the myotome of more mature somites at any stage. Our analysis was focused on nestin expression in the nervous system, and therefore we did not test whether there may be correlates to nestin expression reported in mammalian epidermis, heart, pancreas, kidney, and lung (reviewed in [5]).

During late somitogenesis stages zebrafish nestin is widely expressed throughout the developing nervous system. A similar widespread expression has been reported for comparable stages of mouse development where nestin expression and protein distribution were investigated by in situ hybridization and immunohistochemistry $[1,3]$. Further, 
the comparison of zebrafish nestin expression with GFP expression in nestin promoter-GFP transgenic mice, which has been described in great detail $[4,27,28]$, strengthens the notion of evolutionary conserved roles of Nestin in stem and precursor cell development. With progressive development of the nervous system in zebrafish, like in mouse, nestin expression becomes gradually restricted to regions of the CNS which have previously been identified as zones of proliferating stem and precursor cells: the ventricle walls in the CNS, the ciliary marginal zone in the retina and some scattered cells in the medulla oblongata [16-18].

In the peripheral nervous system nestin is strongly expressed in cells associated with the octaval ganglion, the lateral line ganglia, the facial and trigeminal ganglia, and the ganglia of the nervous vagus. It has been reported for mice that nestin in the PNS is expressed in astrocytes associated with neurons of the cranial ganglia [26], therefore it is likely that the nestin expressing cells associated with zebrafish ganglia are also glia.

The zebrafish brain grows throughout post-embryonic stages as well as in mature adult fish. To analyze nestin expression in the maturing post-embryonic and post-larval brain, we studied the brain of four weeks old zebrafish. In the maturing nervous system nestin expression was detected in all areas, which were recently described as stem cell niches and zones of proliferating precursor cells [18]. In the forebrain, these are: the ventricular zones of the tel- and diencephalon, the ventral habenular nucleus (Hav), the periventricular pretectal nucleus (PP), the thalamic nuclei (TN) including the dorsal thalamic nucleus (DT), and the ventromedial thalamic nucleus (VM). In the mid- and hindbrain, these are: the mesencephalic areas of the torus longitudinalis and optic tectum adjacent to the ventricle, the MHB, proliferative zones in the cerebellum, and ventricular zones of the hindbrain and the spinal cord. In summary, nestin expression in the maturing, postembryonic CNS correlates with the previously described proliferation zones [16-18]. Thus, the expression of the intermediated neurofilament nestin in zebrafish, like in mammals, may serve as a marker for stem cells and proliferating precursors in the developing embryonic nervous system as well as in the adult brain.

\section{Methods \\ Fish}

Zebrafish (AB strain) breeding and maintenance was under standard conditions at $28.5^{\circ} \mathrm{C}$ [29]. Zebrafish embryos ( $A B$ strain) were staged and fixed at the desired developmental stages according to Kimmel et al. [30]. To inhibit pigmentation embryos were incubated in $0,2 \mathrm{mM}$ phenylthiourea (Sigma).

\section{In situ hybridization and histological sections}

Whole-mount in situ hybridization (WISH) was performed as described [31]. A Digoxigenin-labeled antisense mRNA probe for nestin was used. For generation of the nestin probe we amplified a fragment from genomic DNA with primers 5' nes_1F: GTACCAGATGCTAGAGCTGAACCACCGCCTTG; 3' nes_1R: GCATCTGCCTCTTGATCCTCGTGCTCTCCAG. These primers amplify a 704 bp fragment of the second exon of the nestin gene (ENSEMBL gene prediction ENSDAR00000040236), which we cloned it into the pBSII-KS vector. For sectioning, embryos were embedded in a mix of $0,5 \%$ gelatin, $30 \%$ bovine serum albumin, and 20\% saccharose dissolved in PBS. Polymerization was initiated by adding 70 $\mu \mathrm{l}$ of $25 \%$ glutaraldehyde per $1 \mathrm{ml}$, the embryos were oriented, and polymerization completed by addition of another $70 \mu \mathrm{l}$ of $25 \%$ glutaraldehyde. Blocks were mounted with glycergel, and $50 \mu \mathrm{m}$ sections were prepared with a Leica Vibratome.

Double fluorescent whole-mount in situ hybridization (FISH) for nestin and pcna was performed modified from $[32,33]$, which were adapted to zebrafish (Alida Filippi and Wolfgang Driever, unpublished, and (30)). Confocal images were recorded with a Zeiss LSM 5 DUO laser-scanning confocal microscope.

For analysis of nestin expression in the brain of $28 \mathrm{dpf}$ fish, animals were anesthetized with tricaine before they were killed in ice water. The brain was dissected out and fixed in $4 \%$ paraformaldehyde/PBS/0,1\% Triton X-100 over night at $4{ }^{\circ} \mathrm{C}$. In situ hybridization was performed as reported for whole mount 24 hpf zebrafish embryos [31]. After the staining, brains were embedded in 3\% agarose/ PBS and serial sections $(50 \mu \mathrm{m})$ generated using a vibratome.

\section{Abbreviations}

ALLG: anterior lateral line ganglion

ALLN: anterior lateral line nerve

CC: crista cerebellaris

CCemol/gra: corpus cerebelli, molecular, granular layer

CeP: cerebellar plate

Ch: chorda dorsalis

$\mathrm{cm}$ : craniofacial mesenchyme

CMZ: ciliary marginal zone

Cven: commissura ventralis rhombencephali 
D: dorsal telencephalic area

DC: diencephalon

DiV: diencephalic ventricle

DT: dorsal thalamus

DTN: dorsal tegmental nucleus

EG: eminentia granularis

eg: enteric ganglia

eth: ethmoid plate

FB: fin bud

FG: facial ganglion

FP: floor plate

g: gut

GC: griseum centrale

GCL: ganglion cell layer

Ha: habenula

HaV: ventral habenular nucleus

Hc, Hd, Hv: caudal, dorsal, ventral zone of the periventricular hypothalamus

Hi: intermediate hypothalamus

hm: head mesenchyme

LC: locus coeruleus

LCa: locus caudalis cerebelli

LR: lateral recess of the DiV

MHB: midbrain hindbrain boundary

MLF: medial longitudinal fascicle

MO: medulla oblongata

NIII: oculomotor nucleus

NIV: trochlear nucleus
NLV: nucleus lateralis valvulae

NMLF: nucleus of MLF

no: notochord

OB: olfactory bulb

OC: otic capsule

OE: olfactory epithelium

OG: octaval ganglion

OT: optic tract

pc: parachordal cartilage

PGZ: periventricular gray zone of the optic tectum

Po: preoptic region

PPa, PPp: parvocellular preoptic nucleus, anterior, posterior part

PPv: periventricular pretectal nucleus, ventral part

pq: palatoquadrate

Pr: pretectum

PTN: posterior tuberal nucleus

PTv: ventral part of the posterior tuberculum

Ret retina

RV: rhombencephalic ventricle

S: subpallium

SC: spinal cord

SFR: superior reticular formation

som: somites

T: midbrain tegmentum

TC: telencephalon

TelV: telencephalic ventricle

TeO: optic tectum 
$\mathrm{TeV}$ : tectal ventricle

TG: trigeminal ganglion

TL: torus longitudinalis

TLa: torus lateralis

TN: thalamic nuclei

TPp: periventricular nucleus of the posterior tuberculum;

TS: torus semicircularis

Val, Vam: lateral, medial division of valvula cerebelli

Vd, Vv: dorsal, ventral nucleus of ventral telencephalic area

VG: vagal ganglion

VMP: ventral motoneuron

III: oculomotor nerve

VII: facial nerve

VIII: octaval nerve

\section{Authors' contributions}

JM performed all experiments, assembled the figures, did the sequence alignments, and wrote a first draft of the manuscript. WD conceived the study and participated in the experimental design, initially identified zebrafish nestin orthologous sequences, and helped to draft the manuscript. All authors read and approved the final manuscript.

\section{Acknowledgements}

We are grateful to Dr. Jochen Holzschuh, Dr. Soojin Ryu, Dr. Alida Filippi (Freiburg University), and Dr. Ela Knapik (Vanderbilt University) for discussions and help in interpreting the expression patterns; Dr. Soojin Ryu for critical reading of the manuscript; Dr. Alida Filippi for providing the Fluorescent WISH protocol and expertise in confocal imaging. Dr. Annette Neubüser for communicating the embedding protocol for vibratome sections. JM is supported by the DFG Graduiertenkolleg I 104. This study was supported by Deutsche Forschungsgemeinschaft Grant SFB 505-B7.

\section{References}

I. Hockfield S, McKay RD: Identification of major cell classes in the developing mammalian nervous system. J Neurosci I985, 5:3310-28.

2. Lendahl U, Zimmerman LB, McKay RD: CNS stem cells express a new class of intermediate filament protein. Cell 1990 , 60:585-95

3. Dahlstrand J, Lardelli M, Lendahl U: Nestin mRNA expression correlates with the central nervous system progenitor cell state in many, but not all, regions of developing central nervous system. Brain Res Dev Brain Res 1995, 84:109-29.
4. Yamaguchi M, Saito H, Suzuki M, Mori K: Visualization of neurogenesis in the central nervous system using nestin promoterGFP transgenic mice. Neuroreport 2000, I I:199|-6.

5. Wiese C, Rolletschek A, Kania G, Blyszczuk P, Tarasov KV, Tarasova $Y$, Wersto RP, Boheler KR, Wobus AM: Nestin expression - a property of multi-lineage progenitor cells? Cell Mol Life Sci 2004, 6 I:25 I0-22.

6. Zimmerman L, Parr B, Lendahl U, Cunningham M, McKay R, Gavin B, Mann J, Vassileva G, McMahon A: Independent regulatory elements in the nestin gene direct transgene expression to neural stem cells or muscle precursors. Neuron 1994, I 2: I I-24.

7. Selander L, Edlund $\mathrm{H}$ : Nestin is expressed in mesenchymal and not epithelial cells of the developing mouse pancreas. Mech Dev 2002, I 1 3: 189-92.

8. Rauch U, Klotz M, Maas-Omlor S, Wink E, Hansgen A, Hagl C, Holland-Cunz S, Schafer $\mathrm{KH}$ : Expression of intermediate filament proteins and neuronal markers in the human fetal gut. J Histochem Cytochem 2006, 54:39-46.

9. EIShamy WM, Ernfors P: Requirement of neurotrophin-3 for the survival of proliferating trigeminal ganglion progenitor cells. Development 1996, I 22:2405-14.

10. Yang J, Cheng L, Yan Y, Bian W, Tomooka Y, Shiurba R, Jing N: Mouse nestin cDNA cloning and protein expression in the cytoskeleton of transfected cells. Biochim Biophys Acta 200I, | 520:25|-4.

II. Dahlstrand J, Zimmerman LB, McKay RD, Lendahl U: Characterization of the human nestin gene reveals a close evolutionary relationship to neurofilaments. I Cell Sci 1992, I03(Pt 2):589-97.

12. Fischer AJ, Omar G: Transitin, a nestin-related intermediate filament, is expressed by neural progenitors and can be induced in Muller glia in the chicken retina. J Comp Neurol 2005, 484: $1-14$

13. Driever W, Solnica-Krezel L, Abdelilah S, Meyer D, Stemple D Genetic analysis of pattern formation in the zebrafish neural plate. Cold Spring Harb Symp Quant Biol 1997, 62:523-34.

14. Driever W, Stemple D, Schier A, Solnica-Krezel L: Zebrafish: genetic tools for studying vertebrate development. Trends Genet 1994, I 0:152-9.

15. Ryu S, Holzschuh J, Mahler J, Driever W: Genetic analysis of dopaminergic system development in zebrafish. J Neural Transm Suppl 2006:6I-6.

16. Zupanc GK, Hinsch K, Gage FH: Proliferation, migration, neuronal differentiation, and long-term survival of new cells in the adult zebrafish brain. I Comp Neurol 2005, 488:290-319.

17. Adolf B, Chapouton P, Lam CS, Topp S, Tannhauser B, Strahle U, Gotz M, Bally-Cuif L: Conserved and acquired features of adult neurogenesis in the zebrafish telencephalon. Dev Biol 2006, 295:278-93.

18. Grandel H, Kaslin J, Ganz J, Wenzel I, Brand M: Neural stem cells and neurogenesis in the adult zebrafish brain: origin, proliferation dynamics, migration and cell fate. Dev Biol 2006, 295:263-77

19. Wullimann MF, Puelles L, Wicht H: Early postembryonic neural development in the zebrafish: a 3-D reconstruction of forebrain proliferation zones shows their relation to prosomeres. Eur J Morphol 1999, 37:| |7-21

20. Ryu S, Holzschuh J, Erhardt S, Ettl AK, Driever W: Depletion of minichromosome maintenance protein 5 in the zebrafish retina causes cell-cycle defect and apoptosis. Proc Natl Acad Sci USA 2005, 102: 18467-72.

21. Ensembl Genome Browser [http://www.ensembl.org]

22. Perron M, Harris WA: Retinal stem cells in vertebrates. Bioessays 2000, 22:685-8.

23. Raymond PA, Hitchcock PF: Retinal regeneration: common principles but a diversity of mechanisms. Adv Neurol 1997, 72: $171-84$.

24. Geling $A$, Itoh $M$, Tallafuss $A$, Chapouton $P$, Tannhauser $B$, Kuwada JY, Chitnis $A B$, Bally-Cuif L: bHLH transcription factor Her5 links patterning to regional inhibition of neurogenesis at the midbrain-hindbrain boundary. Development 2003, I 30: I 59 |-604.

25. Wullimann MF, Rupp B, Reichert H: Neuroanatomy of the zebrafish brain. In Neuroanatomy of the zebrafish brain Edited by: Wullimann MF, Rupp B, Reichert H. Birkhaeuser Verlag: Basel, Switzerland; 1996:vii+ I44. 
26. Izmiryan A, Cheraud $Y$, Khanamiryan L, Leterrier JF, Federici T, Peltekian E, Moura-Neto V, Paulin D, Li Z, Xue ZG: Different expression of synemin isoforms in glia and neurons during nervous system development. Glia 2006, 54:204-13.

27. Kawaguchi A, Miyata T, Sawamoto K, Takashita N, Murayama A, Akamatsu W, Ogawa M, Okabe M, Tano Y, Goldman SA, et al.: NestinEGFP transgenic mice: visualization of the self-renewal and multipotency of CNS stem cells. Mol Cell Neurosci 200I, I 7:259-73.

28. Mignone JL, Kukekov V, Chiang AS, Steindler D, Enikolopov G: Neural stem and progenitor cells in nestin-GFP transgenic mice. J Comp Neurol 2004, 469:3 I I-24.

29. Westerfield M: The Zebrafish Book. Eugene: University of Oregon Press; 1994.

30. Kimmel CB, Ballard WW, Kimmel SR, Ullmann B, Schilling TF: Stages of embryonic development of the zebrafish. Dev Dyn 1995, 203:253-310.

31. Hauptmann G, Gerster T: Two-color whole-mount in situhybridization to vertebrate and Drosophila embryos. Trends Genet 1994, 10:266.

32. Denkers N, Garcia-Villalba P, Rodesch CK, Nielson KR, Mauch TJ: FISHing for chick genes: Triple-label whole-mount fluorescence in situ hybridization detects simultaneous and overlapping gene expression in avian embryos. Dev Dyn 2004, 229:65।-7.

33. Kosman D, Mizutani CM, Lemons D, Cox WG, McGinnis W, Bier E: Multiplex detection of RNA expression in Drosophila embryos. Science 2004, 305:846.

Publish with Bio Med Central and every scientist can read your work free of charge

"BioMed Central will be the most significant development for disseminating the results of biomedical research in our lifetime. "

Sir Paul Nurse, Cancer Research UK

Your research papers will be:

- available free of charge to the entire biomedical community

- peer reviewed and published immediately upon acceptance

- cited in PubMed and archived on PubMed Central

- yours - you keep the copyright 